\title{
Investigation of the Methods for Tool Wear On-Line Monitoring during the Cutting Process
}

\author{
Hongjiang Chen \\ School of Mechanical and Electrical Engineering, \\ Jiangxi Science \& Technology Normal University
}

\begin{abstract}
This paper is well targeted in researching several researches of various tool wear monitoring methods in domestic and foreign. Five important tool wear methods are deeply investigated and described in detail. Through comparing the advantages and disadvantages of each monitoring method according to practical application, and draw a conclusion that the based on multi-sensors method is considered as the main direction of the development of tool wear monitoring in the near future, which would be a great significance for improving the utilization of machine and reducing the economic losses due to the tool wear.
\end{abstract}

Keywords: tool wear, monitoring method, cutting process.

\section{Introduction}

Tool wear is a common phenomenon in cutting process, which directly affects machine-finishing precision, the efficiency and the economic efficiency. Investigation of tool wear can greatly improve the machine-finishing efficiency, and reduce the processing cost, and have enormous economic effect. Industry statistics indicated that tool failure was the primary factor caused the machine breakdown, which accounted for the shutdown of the $1 / 5$ to $1 / 3$ during the total downtime of the CNC machine tool [1]. Studies showed that if CNC machine tool is equipped with monitoring and detection system, it can reduce $75 \%$ the downtime, and enhance productivity $10 \% \sim 60 \%$, and even raise machine utilization above 50\% [2]. American Kennamtal Corporation's research indicated that if the $\mathrm{CNC}$ system is equipped with tool monitoring system, it can save up to $30 \%$ processing fee [3]. Therefore, it is necessary to research the process in tool condition monitoring technology to prevent the work piece scrap caused by tool failure, and to ensure the CNC machine works with trouble-free operation as long as possible.

Tool wear monitoring methods generally can be divided into direct measurement methods and indirect measurement methods [4-6]. The direct measurement methods include discharge current measurement, optical fiber measurement, micro-structure of coating method, resistance measurement method, ray measurement method, as well as computer image processing method. While the indirect measurement methods use the physical quantities, which are mainly related to the cutting process, such as cutting force, torque, cutting italics machine, work piece geometry, chip shape, noise or vibration intensity. The more popular methods acoustic emission monitoring method and multi-sensors are based on monitoring method [7]. 
The direct method of tool condition monitoring has two obvious shortcomings. On the one hand, it needs to stop CNC machine to detect the tool state that occupancies production time. On the other hand, it can not check the sudden damages during the cutting process, which is subject to a certain restrictions on its use [8].

Therefore, the indirect method has become the mainstream of academics who have studied in domestic and foreign.

\section{The Indirect Method of the Tool Condition Monitoring}

Tool condition monitoring techniques are generally consist of the sensor signal acquisition, signal processing and feature extraction and pattern recognition device. The basic structure of the tool condition monitoring system was presented in Figure 1.

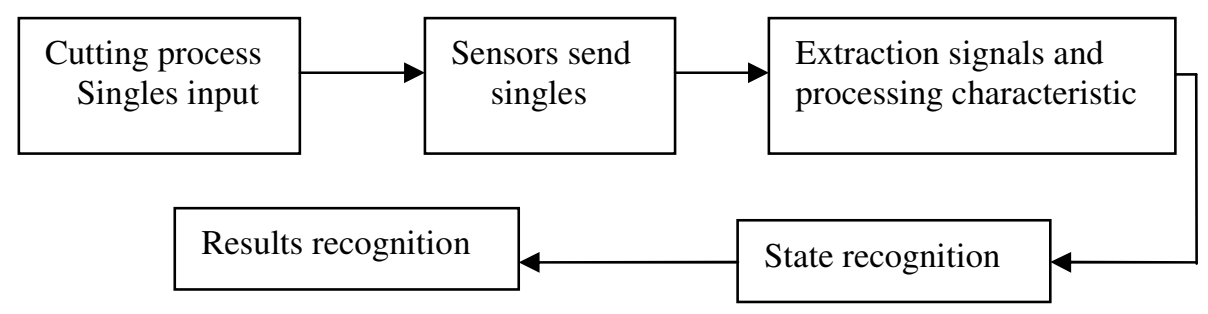

Fig. 1. The basic structure of the tool condition monitoring system

\subsection{Cutting Force Monitoring Method of the Tool Status}

Extensive researches showed that the tool wear of cutting force has a direct inner contact during the cutting process, moreover, the cutting force signals can be accessed through the ordinary resistance strain gauges, or piezoelectric sensors. And the cutting force in general with the increase of tool wear. S. Jetly's experiment results indicated that the feed force is more sensitive than the main cutting force to the tool wear in the turning process [9]. However, Shaohua's [10] research concluded that the main cutting force can reflect the most cutting tool's attrition rate. North China Electric Power University, Kangshi li [11], who designed the system of LABVIEW-based cutting force data acquisition and storage, and its application procedure including the data acquisition and memory of the three cutting force, the cutting force profile demonstration and feedback, the experimental data analysis statistical analysis (error analysis, unusual data processing and so on), and the cutting temperature empirical formula foundation, etc.

Due to the complexity of the cutting process, many factors affect the cutting force, so it is very difficult to establish a precise and perfect cutting force model. Even if has established a perfect cutting force model, it's difficult to distinguish between the changes of cutting parameters caused by the changes of the cutting force component or by the tool breakage ,or by the wear of the cutting pitch changes. In addition, when install the measuring devices required for re-equipping the machine structure, which caused the adjustment and maintenance is inconvenience. Therefore, we can draw the 
conclusion that this method is not suitable for promotion in actual production, especially in some poor condition factories.

\subsection{Cutting Noise Monitoring Method of the Tool Condition}

The measured sound signals contain much information about the state cutting in cutting process, and thus can monitor the tool's state by taking advantage of the sound signals. A. B. Sadat [11], who found that the friction noise's sound level between the tool and the workpiece in the $0.73 \sim 3.5 \mathrm{kHz}$ frequency domain through many experiments by his experimental staff. The blunt tool is approximately $15 \mathrm{~dB}$ higher than the new blade. In the initial wear, the noise sound level increases in an obvious rise, and then stabilized. When the cutting velocity increases, the noise sound level decreased, while the tool cantilever increases, the noise sound level enhanced. L. C. Lee [12] found that in most of the work piece material combinations and operating conditions, there is a cutting noise of the characteristic frequency near 4-6 kHz frequency, its sound pressure level has a very good correlation with the tool wear. Before the rapid wear and tear, the sound pressure level assumes the declining trend.

At present, the cutting noise monitoring method is rarely used in monitoring system. One of the most important reasons is that the voice detection is hard to implement in actual processing workshop, because of the workshop ambient noise is usually about $90 \mathrm{~dB}$. In addition, the real-time signal processing technology, as well as feature extraction method has yet to be further studied. So, the method almost has no application in practice.

\subsection{Acoustic Emission Monitoring Method of the Tool Status}

AE (acoustic emission) technology is a new method for tool wear monitoring in recent years. The AE signal has the transient state and randomness, belongs to the nonsteady random signal. We have done many experiments studies in laboratory, and have discovered that in the metal-cutting process has rich information of AE. The socalled AE phenomenon means that when the solid occurs in deformation or fracture, it emits elastic wave, therefore, AE signal is a mechanical wave in nature, with a wave of basic characteristics of volatility and decaying. In metal-cutting process, the following phenomena can generated AE signals, such as the tool flank of the friction and chip impact, breaking, as well as the plastic deformation of shear zone, the friction of the rake face's second area and so on [14]. When the cutting tool occurs in wear and damage, the acoustic emission signals will be changed. During the monitoring and testing processes, generally use piezoelectric sensors to pick up signals and approach to the cutting tool as far as possible.

At present, the characteristics of acoustic emission monitoring have the RMS value energy analysis, ring count, amplitude distribution and spectral analysis and so on. T. Blum studied the changes in cutting conditions affect the RMS of acoustic emission signals, the count rate, and discovered that the RMS value of acoustic emission signals are increased with the extent of the flank wear of cutting tool, and also increased with the cutting parameters enlarged, there exists a linearly increasing relationship between the count rate and the cutting speed, the total acoustic emission event rate is increased with the feed rate decreased [15]. However, the overall incidence of acoustic emission is increased alone with the feed rate decreased. 
According to the above analyzed, we can draw the conclusion that the advantages of the acoustic emission monitoring tool wear states as follows: firstly, the acoustic emission frequency range is much higher than the mechanical vibration and environmental noise. So, the high-pass filter is very easy to obtain the non-disturbance signals. Secondly, the sensor of receiving acoustic emission piezoelectric crystal can be easily installed in the tools without affecting the process. Thirdly, AE signals can avoid the worst impact zone that the machine processing of low-frequency noise, affected by vibration and sound-frequency noise is very little, a higher signal to noise ratio in the high frequency area, to facilitate the signal processing, and there is little influence on vibration and sound frequency noise [16].Fourthly, the acoustic emission sensor responses quickly with high sensitivity in its working conditions.

Therefore, this method has the good application prospects and is used widely compared with the former two methods nowadays.

\subsection{The Current Monitoring Method of the Tool Status}

Currently, it is a new popular method to utilize the monitoring motor current signals to monitor the tool wear in foreign factories. During the cutting process, if the tool wear and breakage occur, the cutting force correspondingly changes, which would cause changes in motor's output torque, and the motor's power increases along with it, which leads to the corresponding changes in motor current [17]. The current monitoring method achieves an indirect on-line real-time to determine tool wear and breakage by monitoring the motor current change. Based on LABVIEW technology, Nanchang University, Feng Yan [18] and others achieved a collection of the machine spindle motor current signals, storage and analysis. The online monitoring electric current's change situation reflected the status of tool wear, and proposed the mathematical model between the spindle current and cutting parameters. Besides, they applied experiments to verify the effectiveness and practicality of the system.

Through the above investigated and Compared with the method of cutting force, we can get the results that the machine power method has the advantages as follows: On the one hand, the convenience of measuring signals, and can avoid disturbing from cutting chip environment, oil, smoke, vibration and so on, the monitoring device is easy to be installed. On the other hand, through monitoring the motor current to distinguish the changes in tool condition, which has the features of the convenience to extract current signals, and is convenient to install sensors, what is more, it could not be affected by processing environment. Therefore, it is convenient to be applied in production, and now many famous domestic factories are beginning utilize the method to monitoring tools cutting status.

\subsection{Based on Multi-sensors Monitoring Method}

Although each sensor has its own characteristics, it can describe the measurement object only in a certain range from one hand. Due to the influence of external interference noise, and it will have a greater measurement errors sometimes. So there is no any other sensor can guarantee to provide comprehensive, accurate information at any time, this is the deadly fault to single sensor. In fact, single sensor can only provide partial and inaccurate information. In such condition, based on the multiple-sensors 
monitoring method was proposed by many scholars. YanShan University, ZhiYanLiu [19], who used the method of acoustic emission (AE) in combination with the motor current parameters of multi-feature detection, developed the tool breakage monitoring system with its own features. And established a mathematical model, achieved parameters measurement, and experimentally proved that the system on-line monitoring tool breakage is feasible.

After many social investigation and statistics, we discovered a phenomenon that it is very popular to apply the multi-sensors monitoring method in many common domestic and foreign factories, and the main reason is that the multi-sensors data integration has some advantages that we discovered through a further research. And the advantages are as following: Firstly, it could acquire the tested object or environmental information more accurately. What's more, it also could obtain information with higher accuracy and reliability than any other single sensor. Secondly, it could obtain the independent feature information that one single sensor could not through the sensors complement with each other. Thirdly, it could acquire the same information with less time and lower price to compare with the traditional single sensor. Finally, according to the priori knowledge of the system, it could complete tasks of classification, judgment, decisionmaking and so on through processing the integration information of multi-sensors. According to the above research, we can draw a conclusion that this method is considered as the main direction of the development of tool wear monitoring in the near future, which would be a great significance for improving the utilization of machine and reducing the economic losses due to the tool wear.

\section{Conclusions}

The tool wear online monitoring is an important research direction in advanced manufacturing technology, which is of a great significance for improving production efficiency, reducing processing cost and guaranteeing the processing quality. According to investigate, analyze and comparison in this paper, we can conclude that the based on multi-sensors monitoring method system to monitor tool status can effectively improve the discriminate accuracy, and with higher reliability, and is more suitable for actual production. Therefore, this method would be the main direction of the development of tool wear monitoring in the near future.

\section{References}

1. Ji, Y.: Life Prediction of Tool Wear in FMS environment research. Shijiazhuang Vocational and Technical College 17(6), 35-37 (2005)

2. Adam, G., Jin, J., Oban, P.E.: State-of-the-art methodsand results in tool condition monitoring: a review. Int. J. Adv. Manuf. Technol. (26), 693-710 (2005)

3. Feng, Q.g.: Study of the Cutting tool condition online monitoring strategies. Shanghai Jiaotong University master's degree paper (2004)

4. Li, Y.h.: Study of the tool condition with multi-sensors monitoring strategies. Shanghai jiaotong university, PHD paper (1999)

5. Li, Y.h.: Study of the tool condition with multi-sensors monitoring strategies. Shanghai jiaotong university, PHD paper (1999) 
6. Jemielniak, K.: Tool Wear Monitoring Based on a Non-Monotonic Signal Feature. Proceedings of the Institution of Mechanical Engineers, Part B: Journal of Engineering Manufacture 220(2) (2006)

7. Silva, R.G., Wilcox, S.J., Reuben, R.L.: Development of a system for monitoring tool wear using artificial intelligence techniques. Proceedings of the Institution of Mechanical Engineers, 220(8) (2006)

8. Spirgeon, D., Slater, R.A.C.: In process indication of surface roughnessusing a fibre potics transducer. In: Proc. III. 15th Int. Machine Tool Design and Research Cone, pp. 339-347 (1974)

9. Kang, S.L., Yi, S.-M., Lu, Y.: LABVIEW-based cutting force monitoring system. Combination Machine Tool \& Automation Engineering Technology (8) (2004)

10. Shao, H.: Study and development the system of Milling power monitoring tool monitoring system. Shanghai Jiaotong University, PHD paper (1994)

11. Sadat, A.B., Kidd, S.R., Hand, D.P.: Acoustic emission monitoring of tool wear during the face milling of steels and aluminium alloys using a fibre optic sensor Part 2: frequency analysis. Proceedings of the Institution of Mechanical Engineers, Part B: Journal of Engineering Manufacture, 211(4) (1997)

12. Lee, L.C., Karpovich, R.J.: A study of the relationship between remote thermocouple temperatures and tool wear in machining. Int. J. Prod. Res. 25, 129-141 (1977)

13. Chen, x.-q., Li, x.n., Deng, m.: Metal cutting process in the acoustic emission phenomenon and its research significance. Journal of Chang Sha University of Electric Power 16(1), 75-76 (2001)

14. Moriwaki, T., Tobito, M.: A new approach to automatic detection of life of coated tool based on acoustic emission measurement. Trans. ASME. J. Eng. Ind. 112, 212-218 (1990)

15. Blum, T.: Mill breakage of acoustic emission detection technology. Combination machine tool and automation processing technology (3) (1992)

16. Feng, Y., Luo, 1., Xia 1.: LABVIEW-based online monitoring system for tool wear. Sentinel surveillance (12) (2006)

17. Jun, z., Wang, x.: Monitoring.: Motor current to determine the tool wear and breakage. New Technologies (6) (1990)

18. Feng, Y., Luo 1., Xia 1.: LABVIEW-based online monitoring system for tool wear. Sentinel Surveillance (12) (2006)

19. Liu, Z.y., Wang, j.: Using acoustic emission and electric current gun detection technology to achieve the monitoring of tool wear, mechanical, May 2 (1999) 